DESARROLLO DE UN PLAN DE SOCIAL MEDIA PARA POSICIONAR UNA EMPRESA EN CANCÚN, QUINTANA ROO.

\title{
DESARROLLO DE UN PLAN DE SOCIAL MEDIA PARA POSICIONAR UNA EMPRESA EN CANCÚN, QUINTANA ROO
}

\section{DEVELOPMENT OF A SOCIAL MEDIA PLAN TO POSITION AN ORGANIZATION IN CANCUN, QUINTANA ROO}

\begin{abstract}
Lucero del Carmen Mul Canul*, Enrique Roberto Peralta Mazariego**, José Félix García Rodríguez***
\end{abstract}

\begin{abstract}
*Estudiante de Técnico Superior Universitario en Desarrollo de Negocios Área Mercadotecnia. Universidad Tecnológica de Cancún. División Económico Administrativa. Email: luceromul16@gmail.com. ORCID: https://orcid.org/0000-0001-8014-8635.

**Doctor en Administración Educativa. Universidad Tecnológica de Cancún. División Económico Administrativa. Email: mazariego27@hotmail.com. ORCID: https://orcid.org/0000-0001-9197-879X.

*** Doctor en Finanzas Públicas. Universidad Juárez Autónoma de Tabasco. División Académica de Ciencias Económico Administrativas. ORCID: https://orcid.org/0000-0002-7319-1472.
\end{abstract}

Dirección para correspondencia: jfgr55@hotmail.com

Fecha de recibido: 31 de julio de 2019

Fecha de aceptación: 25 de agosto de 2019

DOI: https://doi.org/10.19136/hitos.a25n73.3533

(c) (i) (8)

MUL-CANUL L. DEL C., PERALTA-MAZARIEGO E. R. GARCÍA-RODRÍGUEZ J. F. 
DESARROLLO DE UN PLAN DE SOCIAL MEDIA PARA POSICIONAR UNA EMPRESA EN CANCÚN, QUINTANA ROO.

\section{RESUMEN}

Las estrategias de marketing, tales como: incrementar el número de clientes de la organización, incentivar las ventas, dar a conocer nuevos productos, ampliar la cobertura comercial, entre otras estrategias deberán contar con funciones especializadas, en la cual se desarrollan técnicas y métodos como el uso de las redes sociales, que permitan identificar las prioridades y preferencias.

OBJETIVO: Desarrollar una prueba piloto de un Plan de Social Media, para una página de Facebook de una empresa de Cancún.

MATERIAL Y MÉTODO: Se realizó una investigación con enfoque cuantitativo de tipo descriptivo.

RESULTADOS: Se realizó el Plan de Social Media para Facebook, una selección del tipo de contenido. Se notó el aumento de interacción en la página a partir del inicio de la prueba piloto consiguiendo más "Me gusta y compartidos".

CONCLUSIONES: Un Plan de Social Media necesita de una constante gestión y tiempo para poder notar los resultados favorables en cuanto a las estrategias aplicadas y sobre todo, nunca dejar de lado los pilares fundamentales: creación de contenido y control adecuando y constante.

PALABRAS CLAVE: Social Media. Redes sociales. Marketing. Facebook.

\section{ABSTRACT}

Marketing strategies, such as: increasing the number of clients in the organization, encouraging sales, launching new products, expanding commercial coverage, among other strategies must have specialized functions, through which techniques and methods such as the use of social networks are developed, allowing to identify priorities and preferences.

OBJECTIVE: To develop a pilot test of a Social Media Plan, for a Facebook page of a Company based in Cancún. 
DESARROLLO DE UN PLAN DE SOCIAL MEDIA PARA POSICIONAR UNA EMPRESA EN CANCÚN, QUINTANA ROO.

MATERIAL AND METHOD: An investigation with a quantitative and descriptive approach was carried out.

RESULTS: A Social Media Plan for Facebook with a selection of content type was designed The increase of interactions on the page from the beginning of the pilot test was remarkable, resulting in more "likes and shares".

CONCLUSIONS: A Social Media Plan needs constant management and time in order to notice the favorable results of the applied strategies and, above all, never neglect the fundamental pillars: the creating of content, as well as an adequate and constant control.

KEY WORDS: Social Media. Social networks. Marketing. Facebook.

\section{INTRODUCCIÓN}

Hoy en día, es difícil imaginar a una organización no se encuentran inmersas en el uso masivo y generalizado de las redes sociales, las cuales se han convertido en el canal ideal para llegar a los diferentes segmentos de una manera personal, aunque en esencia el acercamiento se deben a los cambios tecnológicos y el crecimiento de las diversas plataformas sociales. Para el caso de las pequeñas y medianas empresas, se ha convertido en el motor económico en México, por la cantidad de organizaciones que lo integran (Pérez, 2012).

Las estrategias de Marketing están diseñadas para orientar a los gerentes a obtener productos para los consumidores y alentarlos a comprar, identificar las necesidades del cliente, fijar precios, especificar cuando y donde se venderán los productos. Las estrategias de marketing, tales como: incrementar el número de clientes de la organización, incentivar las ventas, dar a conocer nuevos productos, ampliar la cobertura comercial, entre otras estrategias deberán contar con funciones especializadas, en la cual se desarrollan técnicas y métodos como el uso de las redes sociales, que permitan identificar las prioridades y preferencias (Real, Leyva y Heredia, 2014). 
DESARROLLO DE UN PLAN DE SOCIAL MEDIA PARA POSICIONAR UNA EMPRESA EN CANCÚN, QUINTANA ROO.

Algunas de estas redes, como Facebook por ejemplo, son tan utilizadas como plataforma online en diversos entornos, ya sea profesional, personal o comercial. De ahí, que muchas organizaciones deciden crear su cuenta en esta red social para aplicar el marketing, debido a que es un medio de comunicación con muchos beneficios y oportunidades (Peñarroya, 2014).

Por consiguiente, en este trabajo se presenta un análisis de una página de Facebook en cuanto a su número de Likes y seguidores, como parte fundamental la interacción y el alcance que tenían hasta el momento las publicaciones de la plataforma. De esta forma, se plantean los diferentes tipos de contenido a considerar para realizar publicaciones que logren ser llamativas y relevantes para los usuarios.

\section{MARCO TEÓRICO}

\section{Redes sociales}

Cuando se piensa en una red la palabra se asocia a una especie de ensamble, una concatenación de vínculos que une o sirve como puente entre un extremo y otro, entre un punto, sistema, lugar o persona y otro, y su correspondiente antagonista (Velázquez, 2014).

Según Velázquez (2014, p. 68) "la frase redes sociales, refiriéndonos a entornos donde el Internet es el protagonista, proviene de un encadenamiento de entidades ligadas de forma remota entre sí por medio de la tecnología-computadoras, teléfonos celulares. El hecho de poder enviar y recibir información, conectarse- entre un punto (persona, entidad) de la cadena y otro (persona, entidad), convierte por defecto, a este ensamble en social, entendiendo lo social como aquella arena o entorno donde se promueve el interés común y mutuo".

Por su parte, Leal y Quero (2011, p. 294) definen una red social "como un servicio basado en Internet que permite a los individuos construir un perfil público o semi-público dentro de un sistema delimitado, articular una lista de otros usuarios con los que comparten una conexión".

Debido a esto, el número de usuarios en las diferentes redes sociales ha ido en aumento ya que existe una gran variedad de estas, las cuales varían en cuanto a su temática y funcionalidad. Nunca dejando de lado su concepto principal que es permitir a los usuarios comunicarse con otros. 
DESARROLLO DE UN PLAN DE SOCIAL MEDIA PARA POSICIONAR UNA EMPRESA EN CANCÚN, QUINTANA ROO.

Por otro lado, Toledo (2012, p. 5) considera que "las redes sociales, ideadas inicialmente para establecer canales de comunicación exclusivamente entre personas, ha ido poco a poco perfilándose como escenarios útiles para las empresas y marcas".

De esta forma, el panorama de las redes sociales se ha ido ampliando a un terreno que ha sabido conquistar, ya que de esta forma permiten que las marcas reciban a sus clientes o seguidores con los brazos abiertos, debido a que estos se ven interesados por las marcas, basándose así en los principios básicos de la atracción.

Por ello, es que las organizaciones quieren encontrar clientes potenciales en las redes sociales pero son muy pocos los que realmente lo consiguen de una manera efectiva. Para generar cliente potenciales radica en ofrecerles algo valioso, algo que les llame la atención o que por algún motivo les resulte interesante, y para descubrirlo un buen ejercicio es ponerse por unos minutos en la mente de tus clientes (Merodio, 2010, p. 23).

\section{Facebook}

Según Smith (2017, p. 6) Facebook es una de las plataformas de redes sociales más populares, y contempla un $77 \%$ de todos los inicios de sesión en las redes sociales. Además, es un medio excelente para involucrarse con el público, crear autoridad, dirigir tráfico hacia tu sitio web y aumentar tu credibilidad como marca. Por tanto, contribuye a enfocar al público, y a transformar a estas personas en clientes potenciales, creando finalmente una base de clientes leales.

Por otra parte, Gálvez (2015, p. 27) considera que estar presente en esta red social en la que 1.300 millones de usuarios no sólo ven el contenido de la marca, sino que también participan en su difusión, representa un paraíso para aquellas empresas que han integrado el social media dentro de la estrategia de marketing.

Por ello, es relevante entender la importancia de diseñar un buen plan de social media. Cualquier organización que desee actuar en Facebook, debe tener en cuenta que no es tan simple como crear un perfil, exponer los productos y comenzar a vender.

Lecinski (2011, p. 33) realizó un estudio que reveló que el 37\% de los compradores consideran que las fuentes de interacción social en línea ejercen una gran influencia en la toma de sus decisiones; este porcentaje aumentó en comparación con el 19\% en 2010; casi se duplicó en un año. Las principales actividades sociales en línea de los compradores, son:

MUL-CANUL L. DEL C., PERALTA-MAZARIEGO E. R. GARCÍA-RODRÍGUEZ J. F. 
DESARROLLO DE UN PLAN DE SOCIAL MEDIA PARA POSICIONAR UNA EMPRESA EN CANCÚN, QUINTANA ROO.

1. Obtener una referencia en línea de un amigo.

2. Volverse amigo o seguidor de una marca.

3. Leer blogs donde se intercambien opiniones sobre productos.

4. Ver los comentarios en redes sociales como Facebook.

En ese sentido, Facebook es más utilizada por usuarios dentro del grupo de edad de 26 a 35 años. Urueña, Ferrari, Blanco y Valdecasa (2011, p. 31). El 35\% de los "compradores" señala que les gusta más comprar a marcas que les envían correos electrónicos consentidos; el 27\% es más propenso a comprar a marcas después de seguirles en Twitter y un 17\% compra a marcas después de hacerse su fan en Facebook (Urueña, Ferrari, Blanco y Valdecasa, 2011, p. 59).

De acuerdo a Molina (2013, p. 7) para la mayoría de las pymes, los inicios en social media son muy difíciles, sobre todo porque han de construir la comunidad desde prácticamente 0 . Por esta razón, una forma idónea de iniciar una campaña en Facebook es lanzando un concurso (eso sí, depende del tipo de pyme y sus objetivos). Esto permitirá llegar a más clientes, atraerlos y, sobre todo, fidelizar a los que ya se tienen.

El primer paso es tener claro los objetivos. ¿Qué quieres conseguir? ¿Dónde quieres llegar? y elegir entonces qué tipo de concurso es el que más te conviene.

En Facebook puedes lanzar dos tipos de concurso:

1. Los sweepstakes (un juego de lotería): los usuarios simplemente dejan sus respuestas en el sitio creado a tal efecto (respuestas de "sí" o "no" a un post, por ejemplo, o votar por una u otra imagen), y espera a que la suerte le acompañe y su respuesta sea la ganadora.

2. Los contests (competición): requiere más creatividad de los candidatos. Subir una fotografía, un vídeo, un relato, dar una respuesta creativa.

Todo ello, implicará una mayor fidelización del cliente. 
DESARROLLO DE UN PLAN DE SOCIAL MEDIA PARA POSICIONAR UNA EMPRESA EN CANCÚN, QUINTANA ROO.

Por otro lado, Lambrechts (2011, p. 34) menciona que se debe evitar:

1. Utilizar el mismo contenido que en otras redes. Duplicar contenido en todas las redes sociales hará que tus seguidores no encuentren un motivo real por el cuál leerte en uno u otro lugar.

2. No programar todo el contenido. Se debe contemplar factores externos de lo cotidiano para interactuar con los usuarios, las redes sociales están vivas.

3. Evitar publicar demasiada información en un mismo día. No es bueno realizar actualizaciones constantes.

4. Hacer uso pero no abuso. No aburrir a quienes leen intentando vender constantemente. Se debe saber encontrar el equilibrio entre la promoción y el valor agregado que le puedas generar a la comunidad para evitar el spam.

Bajo esta perspectiva, Moreno (2017 pp. 8) menciona que de todas las redes sociales, Facebook continúa liderando el ranking con 2.320 millones de usuarios activos al mes y 1.520 usuarios activos cada día. Por tanto, los clientes potenciales de cualquier marca están en Facebook.

\section{Facebook Ads}

Facebook Ads es la plataforma publicitaria de Facebook, así como online auto gestionable, es decir que permite que cualquier persona pueda crear y gestionar sus campañas publicitarias en Facebook (Moreno, 2017, p. 14).

Por otra parte, Sierra (2016, p. 5) lo define como "el sistema por el cual se puede promocionar una página de Facebook. Se pueden crear anuncios de texto, gráficos y de video, que se mostrarán en el inicio, perfil y fotos de los usuarios, pagando solamente por los clics recibidos, interacciones o por el número de veces que se mostró a los usuarios (impresiones).

Para Gamboa (2017, p. 14) el punto más fuerte de Facebook Ads es la híper-segmentación. Nadie podrá localizar mejor a la persona más interesada en tu nicho que Facebook, basándose en toda la información que obtienen cada día de todos nosotros en su red social; conocen los deseos, preferencias y detalles de cada uno de sus usuarios. 
DESARROLLO DE UN PLAN DE SOCIAL MEDIA PARA POSICIONAR UNA EMPRESA EN CANCÚN, QUINTANA ROO.

De Benito (2016, p. 15) menciona que el modelo de negocio de Facebook es por la publicidad. Facebook ayuda a los anunciantes a conseguir clientes, ayuda a los usuarios a que encuentren buenas oportunidades sin cobrarles por nada.

Por lo tanto, Moreno (2017, pp. 17-18) expone que en Facebook Ads se pueden crear diferentes campañas, según los siguientes objetivos: 1) Promocionar una marca (acciones de branding), 2) Promocionar tu página de Facebook, 3) Clics en el sitio web o tráfico web, 4) Conversiones en el sitio web, 5) Instalaciones de aplicaciones, 6) Interacción con tu aplicación, 7) Llegar a personas que están cera de tu negocio, 8) Interacción con una publicación, 9) Incrementar el número de fans, 10) Respuestas a eventos, 11) Realizar ofertas o promociones especiales y 12) Reproducciones de videos.

\section{Conceptualización del marketing de contenidos}

De acuerdo a Wilcock (2013, p. 5) en la última década han aparecido tres conceptos relacionados, mediante los cuales los profesionales del marketing online han intentado implementar una forma de Marketing, donde la provisión de contenidos promueva directamente un incremento de la confianza en la empresa. Estos tres conceptos: Marketing de contenidos, Inbound marketing y Marketing de permiso, están basados en la idea de fomentar, a través de contenidos de calidad, la atracción de los consumidores hacia la marca, en lugar de acercar la marca hacia los consumidores de una manera más invasiva a través de las campañas tradicionales.

De acuerdo con Sanagustín (2013, p. 6) considera que "el marketing de contenidos va más allá de intentar vender algo al usuario, ya que lo que intenta es establecer una relación con él".

Del Santo (2012, pp. 10) subraya que "el Inbound marketing "se basa en la atracción, es el consumidor el que llega al producto o servicio en Internet o las redes sociales, atraído por un mensaje o contenidos de calidad y obviamente de su interés".

El marketing de permiso, se construye sobre el deber de los responsables de la estrategia de marketing de solicitar permiso a los clientes o potenciales clientes a la hora de ejecutar sus acciones, de esta forma no solo se fomenta un compromiso con los productos y servicios de una forma más cualificada, sino que se obtiene una mayor capacidad a la hora de dirigirnos de 
DESARROLLO DE UN PLAN DE SOCIAL MEDIA PARA POSICIONAR UNA EMPRESA EN CANCÚN, QUINTANA ROO.

manera directa y personalizada a nuestra audiencia mediante el uso de la información proporcionada por ésta de manera voluntaria (Wilcock, 2013, p. 5).

\section{Plan de social media marketing}

El plan de marketing, es la herramienta básica de gestión que debe utilizar toda organización orientada al mercado donde quiera ser competitivo. En su puesta en marcha quedarán fijadas las diferentes actuaciones que deben realizarse en el área del marketing, para alcanzar los objetivos marcados. Este no se puede considerar de forma aislada dentro de la compañía, sino totalmente coordinado y congruente con el plan estratégico, siendo necesario realizar las correspondientes adaptaciones con respecto al plan general de la empresa, ya que es la única manera de dar respuesta válida a las necesidades y temas planteados (Fundación Íntegra de Murcia, 2014).

En concordancia con lo anterior Rojas y Redondo (2013, p. 10) mencionan que el plan de social media "es un conjunto de aspectos que se deben tomar en cuenta para la puesta en marcha de una estrategia de integración de redes sociales y acciones de marketing digital por parte de una empresa".

De acuerdo con Gálvez (2015, p. 28) las diferentes fases que forman parte del plan de social media son:

1. Análisis de la situación y conceptualización: Consiste en un estudio de todo aquello que rodea a la empresa y a la propia organización que nos permita descubrir todos aquellos elementos que pueden llegar a influenciar positiva o negativamente sobre el desarrollo del plan de social media.

2. Definición de objetivos: Se trata de las metas que pretenden alcanzar con la definición puesta en marcha del social medio plan en la empresa. Deben ser optimistas, pero a la vez realistas.

3. Targets y stakeholders: Son los públicos objetivos sobre los cuales se pretende lanzar la campaña para el cumplimiento de los objetivos. Este proceso también es conocido como segmentación de mercado.

4. Contenido: Es el producto estrella de los social media, ya que ofrecer un punto de información relevante a los usuarios supone crear un flujo de comunicación que apunte 
DESARROLLO DE UN PLAN DE SOCIAL MEDIA PARA POSICIONAR UNA EMPRESA EN CANCÚN, QUINTANA ROO.

directamente hacia la fidelización del cliente, en un espacio donde el usuario puede acudir voluntariamente para buscar datos o información.

5. Herramientas y recursos: Son los medios que nos van a permitir alcanzar los públicos objetivos; en este caso, Facebook sería una herramienta del social media plan, el lugar o el canal sobre el cual se desarrollarán las diferentes acciones.

6. Acciones: Son eventos organizados, como concursos o premios, y suponen la puesta en marcha real del plan. En definitiva, responde a la pregunta de qué se va a hacer.

7. Medición y control: Constituye una de las fases más importantes, ya que medir nos permite conocer si se han alcanzado los objetivos predefinidos y tomar decisiones oportunas para futuras acciones.

Para poner en marcha estas estrategias, es necesario asignar a un responsable, que será aquella persona que hará posible llegar a los objetivos, al darle seguimiento y el control necesario, en muchas organizaciones al responsable se le denomina community manager.

\section{Community manager}

En la actualidad, el responsable de ejecutar los planes de social media es conocido como "Community Manager", sin embargo también se considera Social Media Manager a aquella persona responsable de la gestión en el ámbito digital.

Tal como menciona, Del Santo (2012, p. 65) "el community manager es el gestor de las redes sociales. De hecho, eventualmente la labor fundamental de estos profesionales será la de 'gestionar' una comunidad".

Por otra parte, Marquina (2013, p. 15) lo define como "un profesional que se encarga de las relaciones e interacciones entre una entidad y sus usuarios, clientes y público en general a través de los medios y redes sociales online".

\section{Costumer Relationship Management (CRM)}

De acuerdo con Roldan (2016) el CRM es la herramienta de relación de los clientes con las marcas, los productos, servicios y las organizaciones, que están empezando a convivir en un ecosistema digital basado en el diálogo social, usando una red social como Facebook.

En el enfoque de la social media, prevalecen cuatro elementos heredados de la filosofía CRM: 
DESARROLLO DE UN PLAN DE SOCIAL MEDIA PARA POSICIONAR UNA EMPRESA EN CANCÚN, QUINTANA ROO.

a. Inteligencia de clientes: Se necesita tener conocimiento sobre el cliente para poder desarrollar productos y servicios enfocados a sus expectativas.

b. Interactividad: El proceso de comunicación pasa de un monólogo a un diálogo- entre la organización y el cliente-. Además, es el cliente el que dirige el diálogo y decide cuándo empieza y cuando acaba.

c. Fidelización de clientes: En la práctica, es mucho mejor y más rentable fidelizar a los clientes que adquirir clientes nuevos. La fidelización de los clientes pasa a ser más importante y, por tanto, se convierte en el pilar de la gestión de marketing relacional en función del ciclo de vida del cliente. El community manager debe considerar la manera como se establece fidelización proponiendo y utilizando las herramientas de social media marketing.

d. Personalización: Cada cliente desea recibir promociones y ofertas personalizadas, por lo que se necesitan grandes esfuerzos en inteligencia de las bases de datos para desarrollar una eficiente segmentación de clientes.

Tal como menciona, Valle (2015, pp. 14-15) el CRM se compone de tres elementos 1) colaborativo, 2) operacional y 3) analítico, que junto con un buen software de CRM Data Mining, cuya función es analizar los datos que se recogieron en las diferentes partes de la organización, definiendo las previsiones y la forma en que la empresa debe seguir, resulta en un aprovechamiento al máximo de toda la información obtenida para un excelente desempeño de la empresa en relación con todo lo que implica el cliente.

1. CRM colaborativo: Comprende los canales convencionales de comunicación directa 0 indirecta con la empresa, pudiendo ser mantenido por el cliente o por la empresa. Puede ser representado por teléfono, fax, carta, correo electrónico, página web y cara a cara.

2. CRM operacional: Es aquel que tiene la función de mantener y controlar el contacto directo de la empresa con el cliente. También llamado "interacción con el cliente".

3. CRM analítico: Se ejecuta sobre la base de los datos contenidos en las bases de gestión de la empresa con aplicaciones de decisión. Su función es analizar los datos recogidos por las diversas fuentes y generar ensayos con esta información definiendo previsiones y tendencias. 
DESARROLLO DE UN PLAN DE SOCIAL MEDIA PARA POSICIONAR UNA EMPRESA EN CANCÚN, QUINTANA ROO.

\section{OBJETIVO}

Desarrollar una prueba piloto de un plan de social media para una página de Facebook de una empresa de Cancún, la cual debido a una falta de gestión de contenido relevante y llamativo para los usuarios carece de interacción, esto en concordancia con el posicionamiento de esta. La finalidad de realizar la prueba piloto es, demostrar a la empresa los beneficios y ventajas que tiene invertir en un plan de social media. Por el tipo de investigación el alcance se limita a la misma empresa, sin embargo podrá servir como antecedentes para otras PyMEs en la región.

\section{MATERIAL Y MÉTODO}

Se realizó una investigación con enfoque cuantitativo de tipo descriptivo, respaldada en una revisión a profundidad de la literatura, utilizando las fuentes primarias y secundarias de investigación, estas auxiliaron para obtener información segura y necesaria por medio de fuentes bibliográficas, para conocer ciertos conceptos, definiciones y estrategias relacionadas a la social media. La información se recopiló a través de los sitios web de importantes base de datos, como son: Dialnet, Redalyc y e-libro, también se consultaron libros en la biblioteca universitaria y blogs sobre mercadotecnia.

En este proyecto, participó una empresa de la ciudad de Cancún, la cual proporcionó su página de Facebook, así como algunos datos confidenciales para implementarlo. Realizando un análisis de su página de Facebook en cuanto a su número de Likes, seguidores, su interacción y el alcance que tenían hasta el momento las publicaciones de la plataforma.

Se desarrolla una prueba piloto para conocer los resultados de aceptación del contenido por parte de los usuarios, así como por medio de las métricas que Facebook arroja, conocer las métricas que se obtuvieron de acuerdo a la prueba realizada en cuanto al número de Likes y seguidores, así como de la interacción y del alcance las publicaciones, recalcando que los resultados obtenidos fueron de manera orgánica y por medio de pago.

Los beneficios esperados son los siguientes:

- Ahorro en el costo de personal o agencia de mercadotecnia que desarrolle un plan de social media.

- Demostrar los beneficios de la adecuada implementación de un plan de social media.

- Vinculación del sector productivo con el académico. MUL-CANUL L. DEL C., PERALTA-MAZARIEGO E. R. GARCÍA-RODRÍGUEZ J. F. 
DESARROLLO DE UN PLAN DE SOCIAL MEDIA PARA POSICIONAR UNA EMPRESA EN CANCÚN, QUINTANA ROO.

- Desarrollo profesional en proyectos reales y aplicados de los estudiantes.

- Dejar un antecedente para futuros estudios en social media

\section{RESULTADOS}

Se realizó el Plan de Social Media para Facebook, como primera actividad se hizo una selección del tipo de contenido. El tipo de contenido que ofrece una página de Facebook siempre deberá ir acorde al tema principal de esta, para este caso es la comida que ofrecen los diferentes establecimientos o restaurantes en Cancún, sin embargo se consideraron diversos tipos de contenido quedando de la siguiente forma:

- Cómico: memes relacionados con la comida (imágenes, gifs y videos)

- Datos interesantes: temas relacionados con la gastronomía en México, historia y conceptos adquiridos en la actualidad así como la prohibición de uso de plástico y unicel en establecimientos.

- Videos: en los que se presenten recetas para la elaboración de platillos o postres de manera fácil y rápida.

- Encuestas: realizadas para saber dentro de una categoría por ejemplo: hamburguesas, de dos opciones diferentes saber cuáles prefieren, incentivando a que si su preferencia no está dentro de la encuesta puedan dejarla en los comentarios.

- Recomendaciones del día: publicaciones diarias sobre un lugar diferente el cual visitar en Cancún, el contenido puede variar durante la semana: cafeterías, bares, restaurantes, comida rápida, cortes de carne, entre otros.

- Publicidad a establecimientos: básicamente acudir a un establecimiento de comida y realizar un video en el que se promocione el lugar o su "temática" así como alguno de sus platillos, de igual manera acudir a inauguraciones y tomar fotografías para denotar que se está pendiente de las nuevas aperturas.

- Dinámicas: realizarlas en fechas especiales, buscando la colaboración de restaurantes que quieran hacer descuentos en el consumo en su restaurante o regalar una cena para 1 o 2 personas.

La segunda actividad fue realizar la plantilla para el calendario de contenido (ver figura 1) elaborado con la finalidad de tener una mejor gestión en cuanto a las publicaciones de la página de Facebook. 
DESARROLLO DE UN PLAN DE SOCIAL MEDIA PARA POSICIONAR UNA EMPRESA EN CANCÚN, QUINTANA ROO.

\begin{tabular}{|c|c|c|c|c|c|c|c|c|}
\hline \multicolumn{9}{|c|}{ CALENDARIO DE CONTENIDO } \\
\hline Red soclal & Mes: & Año: & & & & & & \\
\hline \multirow[t]{6}{*}{ Fecha: } & HashTag a utilizar & $\begin{array}{l}\text { Frecuencia } \\
\text { de Post }\end{array}$ & $\begin{array}{l}\text { Fechas } \\
\text { Especiales }\end{array}$ & Hora & Objetivo del mensaje & Tema & $\begin{array}{l}\text { Descripción visual de } \\
\text { contenido (De qué se } \\
\text { trata la foto, a dónde nos } \\
\text { lleva el link del post). }\end{array}$ & Mensaje \\
\hline & & & & & & & & \\
\hline & & & & & & & & \\
\hline & & & & & & & & \\
\hline & & & & & & & & \\
\hline & & & & & & & & \\
\hline
\end{tabular}

Figura 1. Plantilla para calendario de contenido para Facebook.

Fuente: Elaboración propia.

Por último, se llevó a cabo una prueba piloto para conocer la aceptación del contenido por parte de los usuarios y conocer por medio de las métricas si las publicaciones tendrían un impacto favorecedor para la página de Facebook. En la figura 2, se presenta una vista previa de las publicaciones realizadas con su respectivo rendimiento (personas alcanzadas, reacciones, comentarios, veces que se compartió):
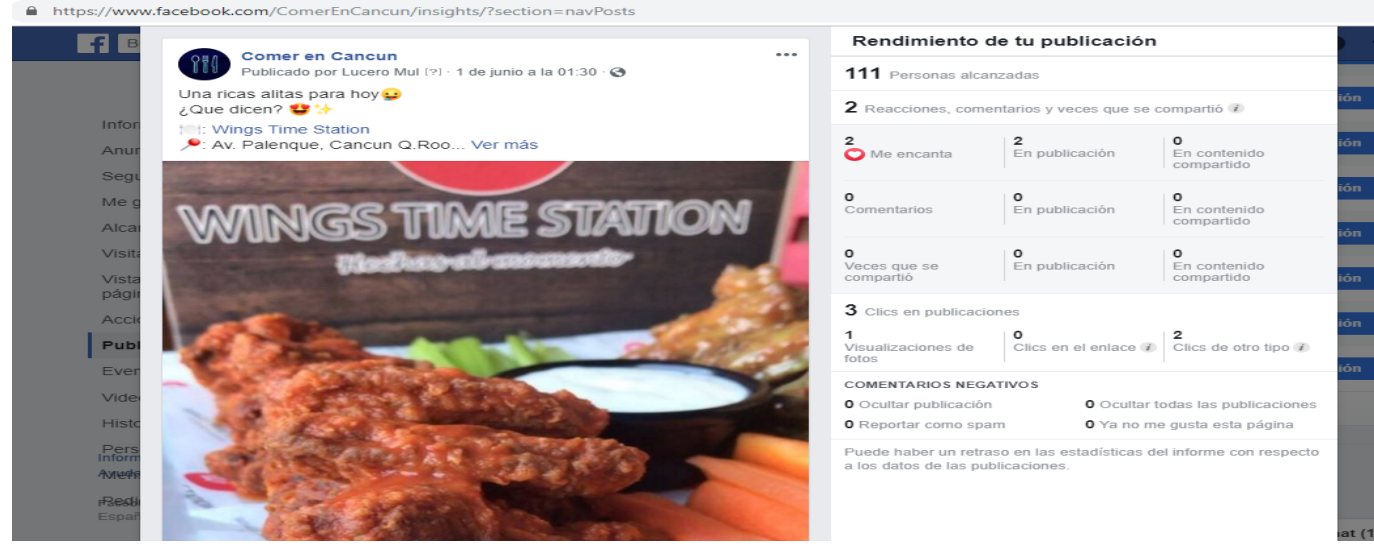

Figura 2. Publicación de recomendación en la página de Facebook.

Fuente: Elaboración propia de las métricas. 
DESARROLLO DE UN PLAN DE SOCIAL MEDIA PARA POSICIONAR UNA EMPRESA EN CANCÚN, QUINTANA ROO.

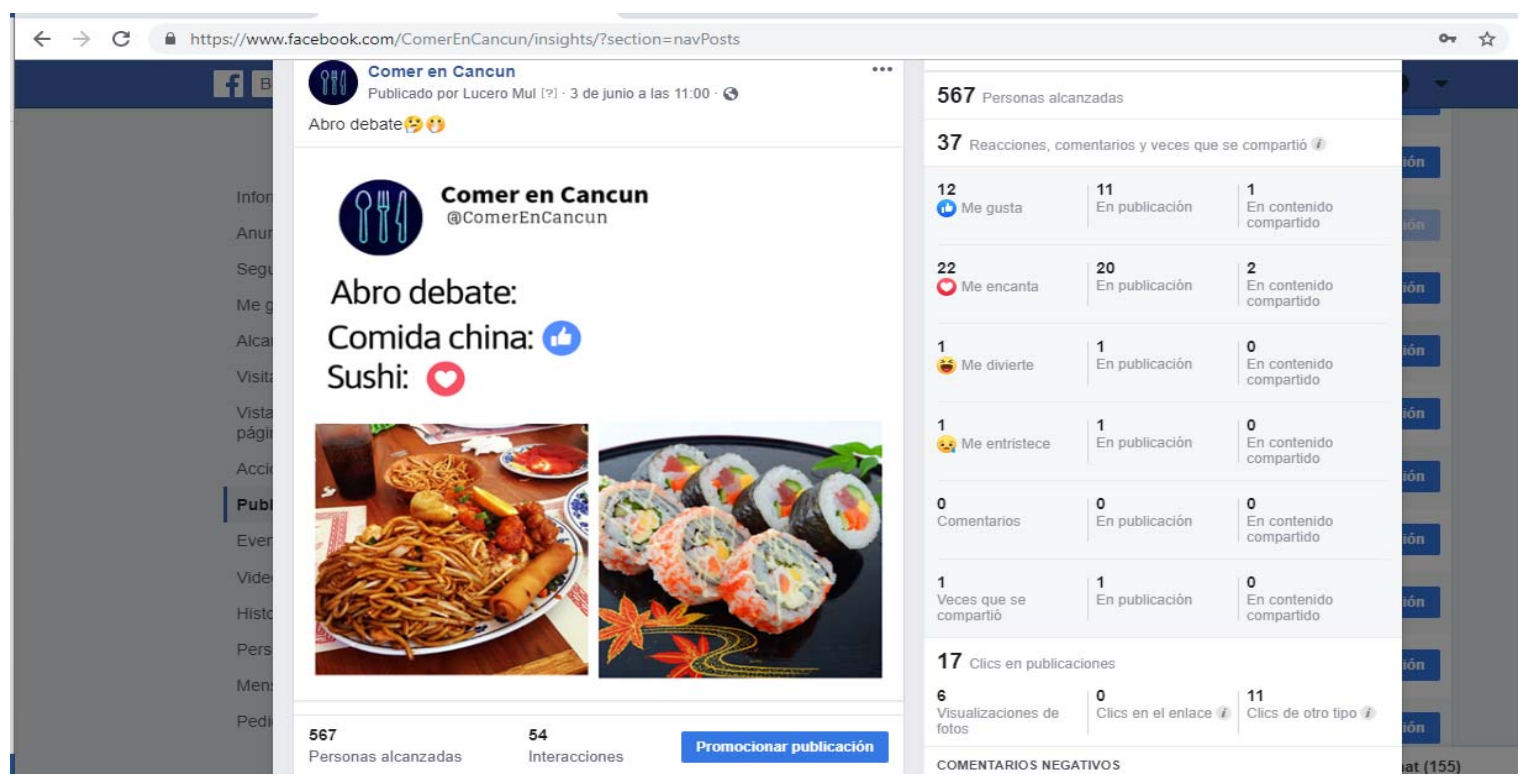

Figura 3. Publicación de debate interactivo en la página de Facebook.

Fuente: Elaboración propia de las métricas.

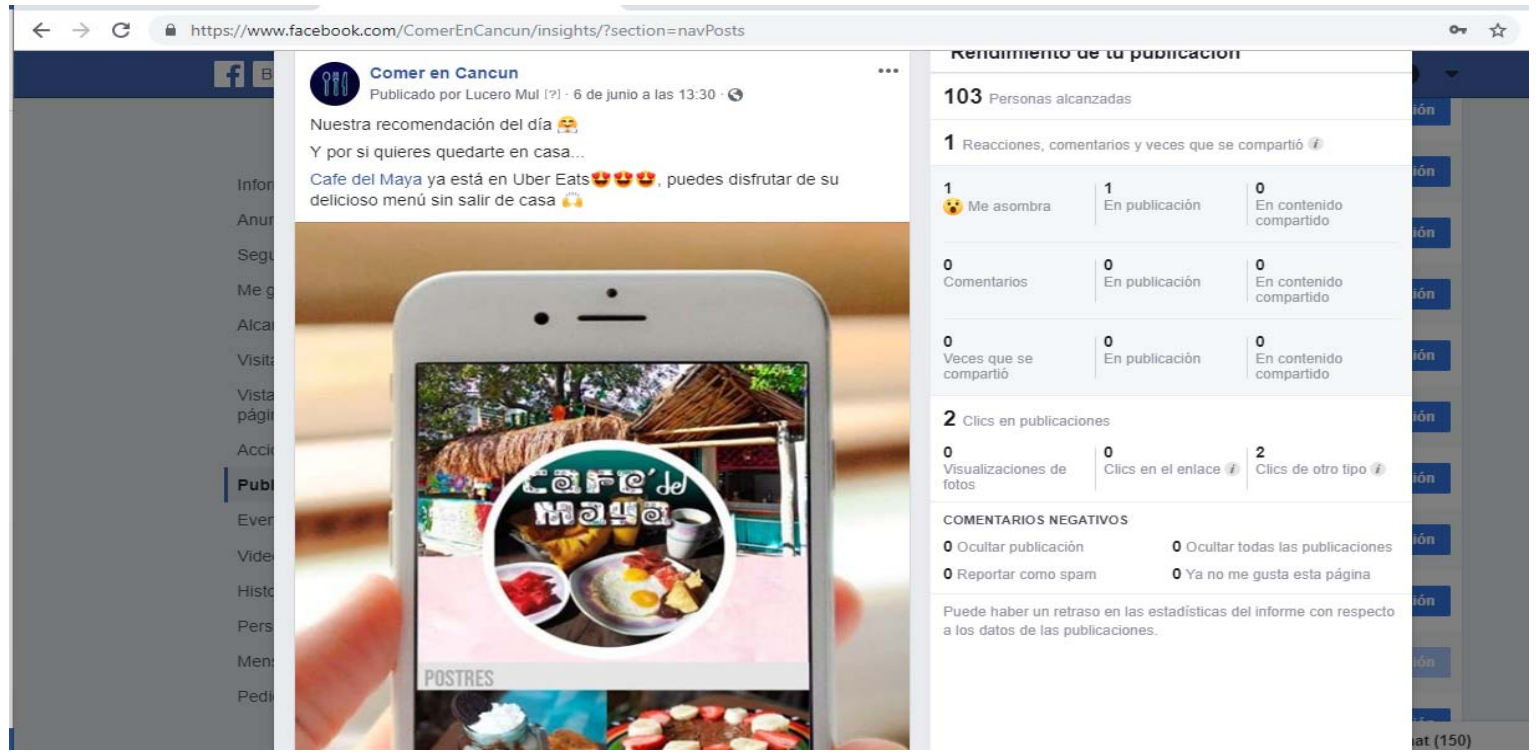

Figura 4. Publicación de publicidad en la página de Facebook.

Fuente: Elaboración propia de las métricas.

Acorde los resultados de la prueba piloto realizada en el periodo del 1 al 15 de Julio del 2019, se pudo destacar el contenido que los usuarios prefieren ver y con el cual interactuar en la plataforma. De igual forma, se pudo añadir contenido más atractivo ya que se dejó de repostear el contenido de otras páginas, además de que las publicaciones ahora tendrían como objetivo el aumento de interacción.

MUL-CANUL L. DEL C., PERALTA-MAZARIEGO E. R. GARCÍA-RODRÍGUEZ J. F. 
DESARROLLO DE UN PLAN DE SOCIAL MEDIA PARA POSICIONAR UNA EMPRESA EN CANCÚN, QUINTANA ROO.

Por otra parte, Facebook realiza una lista de las páginas que estén en constante actualización dejando en últimos resultados a los que están en inactividad; por ello, la página de Facebook tiene un mejor posicionamiento debido a la prueba piloto realizada.

A continuación, se presentan algunas de las estadísticas de la página de Facebook, las cuales sirven como evaluación. De acuerdo con las figuras 5 y 6, el aumento de "Me gustas" en la página a partir del inicio de la prueba piloto el 1 de Junio ha sido notorio, consiguiendo un total de 42 nuevos "Me gusta".

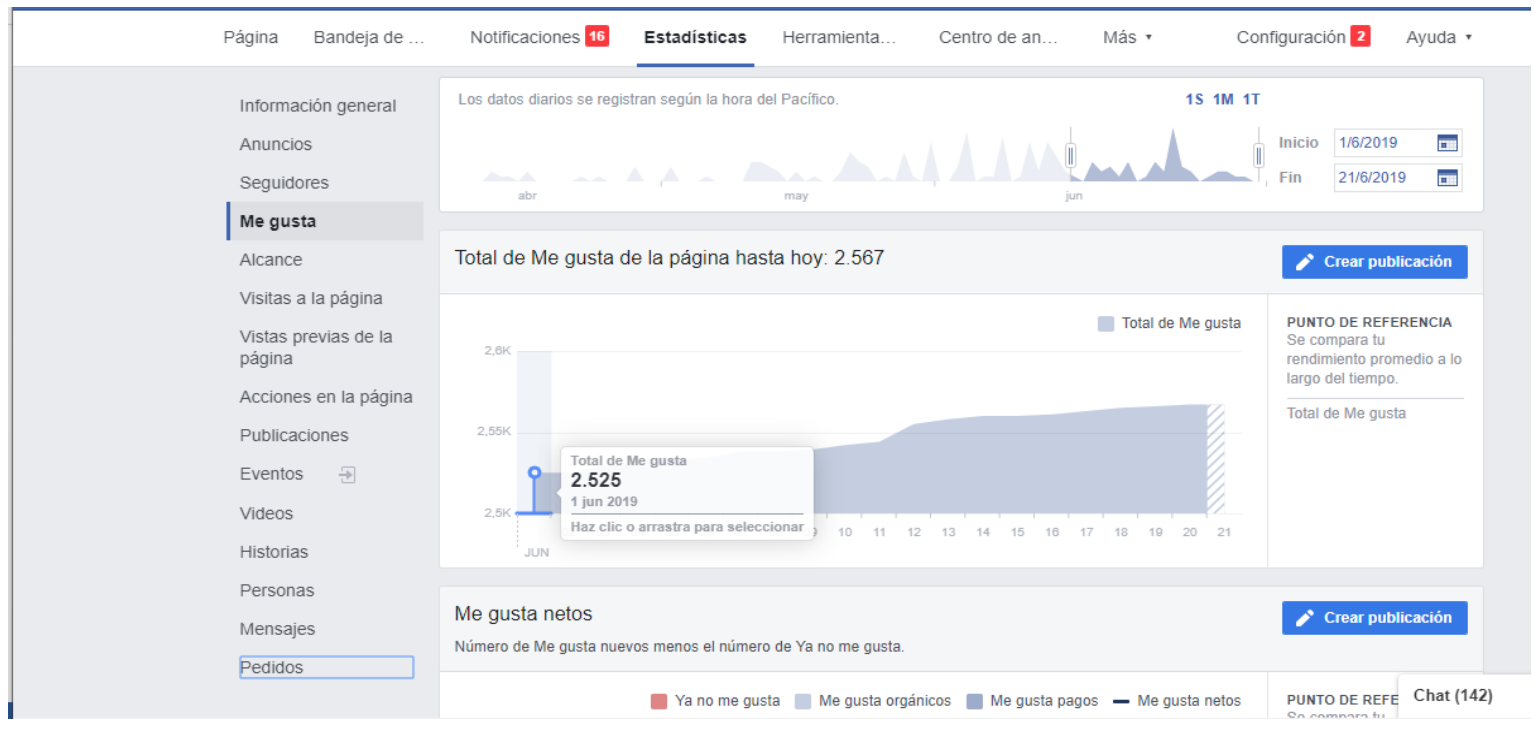

Figura 5. Estadísticas generadas en la página de Facebook al 21 de junio.

Fuente: Elaboración propia de las métricas.

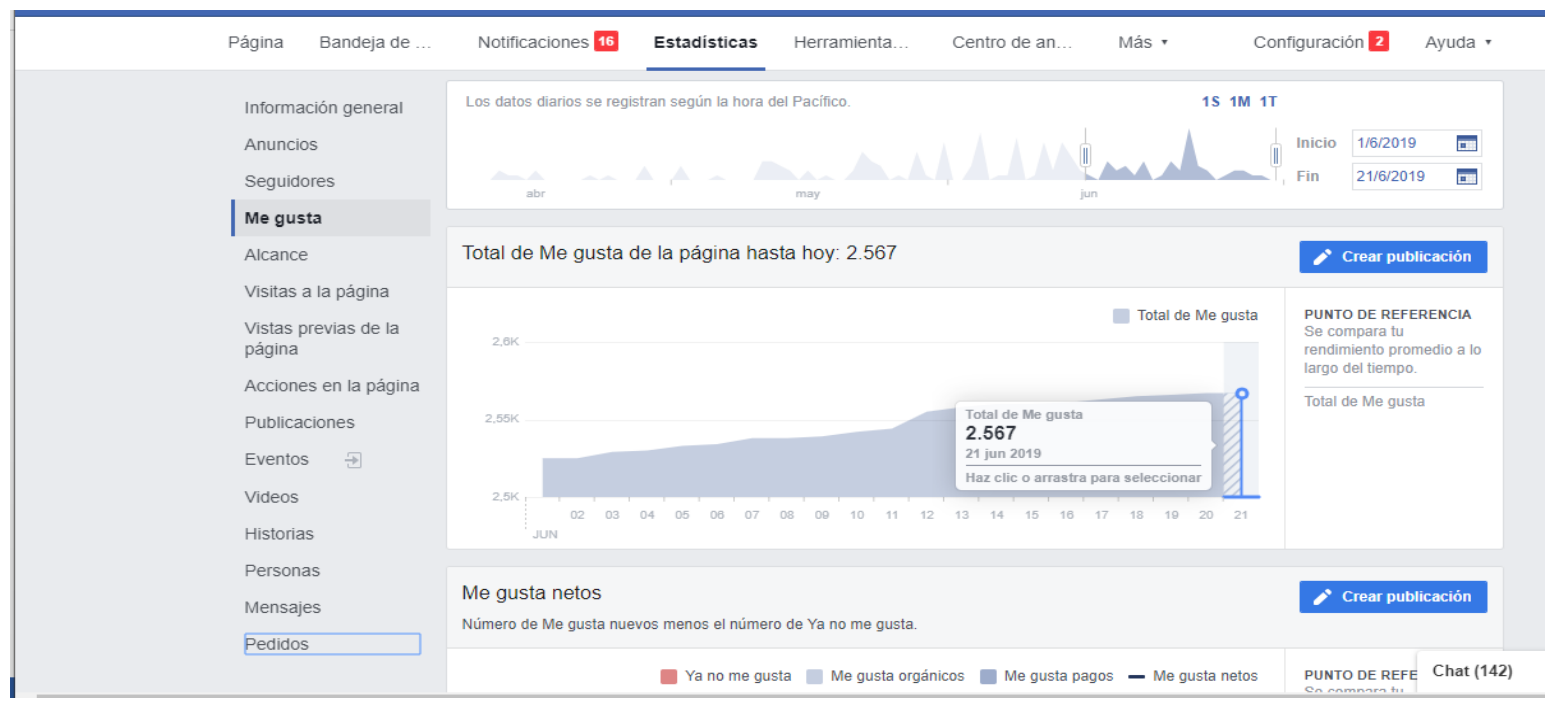

Figura 6. Estadísticas generadas en la página de Facebook al 21 de junio.

Fuente: Elaboración propia de las métricas.

MUL-CANUL L. DEL C., PERALTA-MAZARIEGO E. R. GARCÍA-RODRÍGUEZ J. F. 
DESARROLLO DE UN PLAN DE SOCIAL MEDIA PARA POSICIONAR UNA EMPRESA EN CANCÚN, QUINTANA ROO.

En la figura 7, se puede observar el alcance de las publicaciones realizadas en la prueba piloto, la cual fue en aumento, de tal forma que una de estas publicaciones consiguió tener un alcance de 1,284 personas de manera orgánica.

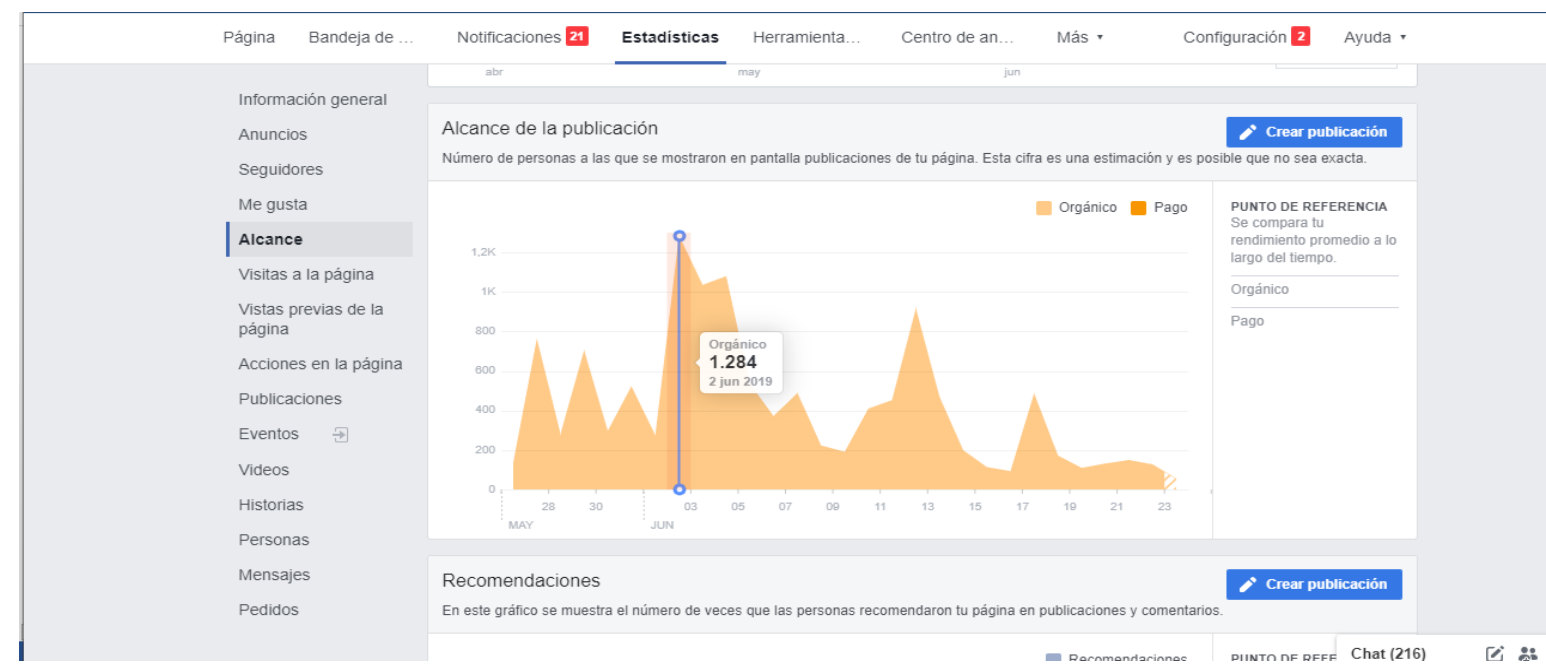

Figura 7. Estadísticas generadas en la página de Facebook al 21 de junio.

Fuente: Elaboración propia de las métricas.

A partir de dicha publicación, el alcance de las publicaciones se mantuvo constante, teniendo como mínimo un alcance de 90 personas de manera orgánica, tal como lo muestra la figura 8.

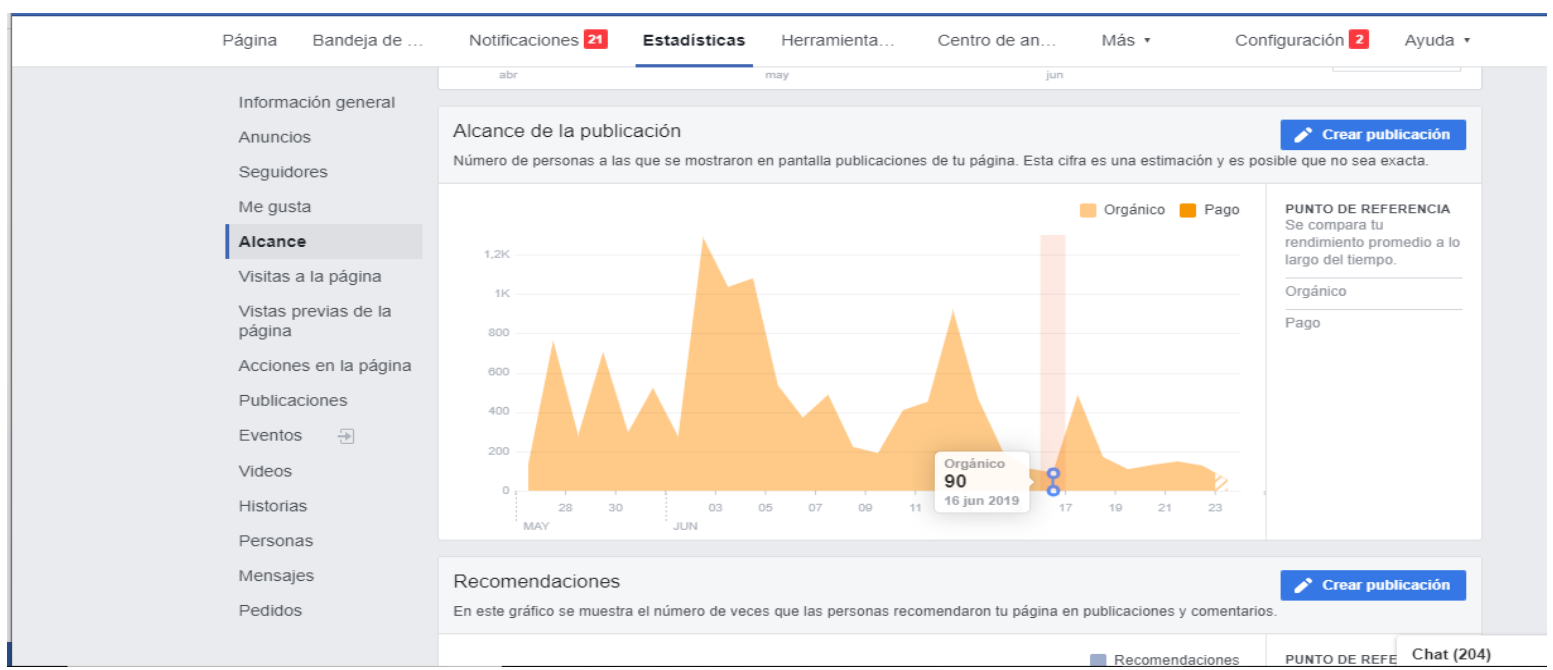

Figura 8. Estadísticas generadas en la página de Facebook al 21 de junio.

Fuente: Elaboración propia de las métricas.

Por último, se debe destacar la interacción (reacciones, comentarios, contenido compartido) que los usuarios tuvieron con las publicaciones, de acuerdo a la figura 9, el número más alto dentro 
DESARROLLO DE UN PLAN DE SOCIAL MEDIA PARA POSICIONAR UNA EMPRESA EN CANCÚN, QUINTANA ROO.

de las publicaciones de la prueba piloto fue de 54 reacciones y de acorde a esta las reacciones fueron constantes.

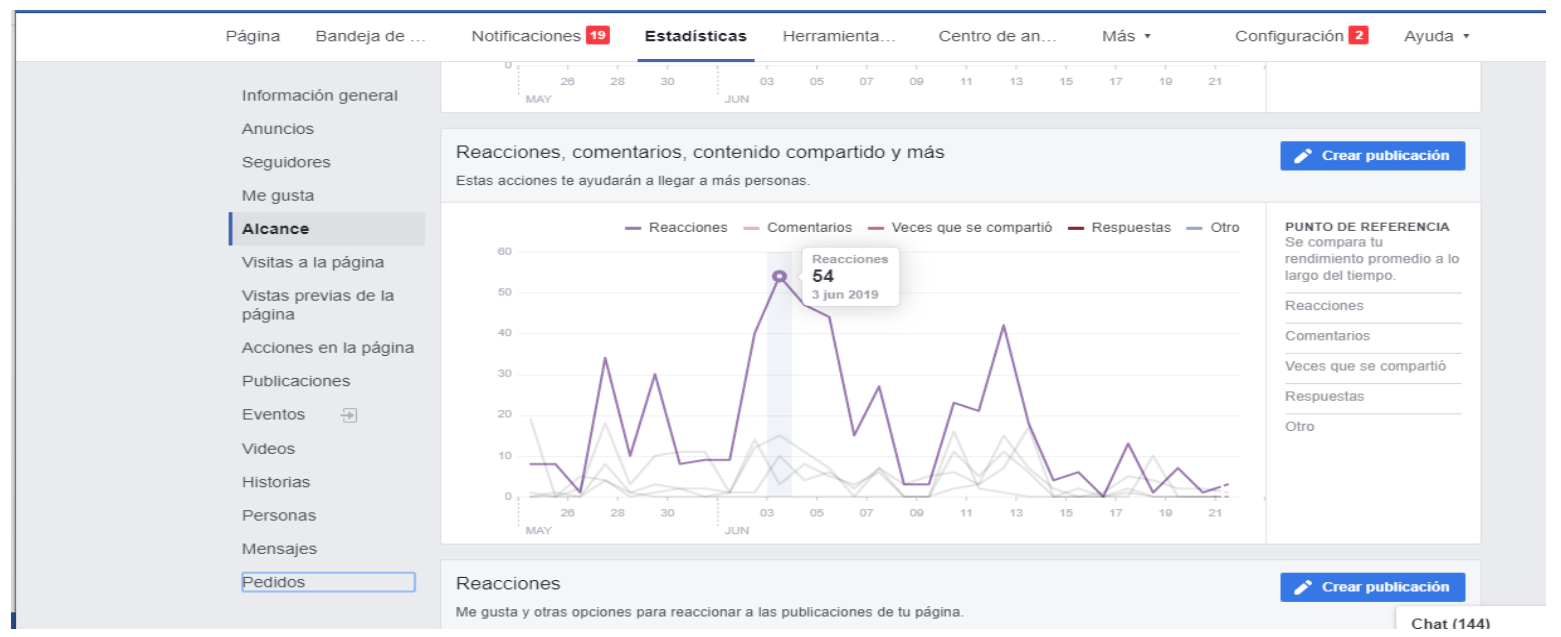

Figura 9. Estadísticas generadas en la página de Facebook al 21 de junio.

Fuente: Elaboración propia de las métricas.

\section{CONCLUSIONES}

El plan de social media es, un conjunto de aspectos que se deben tomar en cuenta para la puesta en marcha de una estrategia de integración de redes sociales y acciones de marketing digital por parte de una organización.

Por tanto, este proyecto tuvo como resultado un plan de social media detallado que tiene como objetivo principal la correcta gestión de una página de Facebook, así como la selección de contenido relevante que denote presencia e identidad, de igual forma conseguir posicionamiento en la mente de los usuarios y sea relevante al momento de buscar algo relacionado con esta.

Para dicho plan, se tomaron en cuenta diferentes aspectos, tales como: la competencia, la situación en la que se encontraba la página de Facebook, la selección de contenido acorde al tema principal de la plataforma, de forma que sea llamativo y se dejar de repostear el contenido de otras páginas y la plantilla a utilizar para elaborar el calendario, la cual fue diseñada para su fácil comprensión, de forma clara y concisa. 
DESARROLLO DE UN PLAN DE SOCIAL MEDIA PARA POSICIONAR UNA EMPRESA EN CANCÚN, QUINTANA ROO.

Tal como se pudo apreciar en la prueba piloto realizada del 1 al 15 de Julio de 2019, Facebook obtuvo estadísticas con resultados positivos para la plataforma, lo cual denota que el contenido publicado fue de agrado a los usuarios, ya que se obtuvo un aumento de 42 nuevos Likes, interacción máxima de 54 reacciones (likes, comentarios) y un alcance máximo orgánico de 1,284 personas.

Se concluye, que un plan de social media necesita de una constante gestión y tiempo para poder notar los resultados favorables en cuanto a las estrategias aplicadas y sobre todo nunca dejar de lado los pilares fundamentales: creación de contenido y control adecuando y constante. De igual forma se recomienda a las organizaciones aplicar pruebas piloto de su plan para ver el comportamiento de la comunidad como se demostró en este proyecto.

Este trabajo, deja como precedente la importancia de aplicar pruebas piloto al momento de gestionar un plan de social media y así, sea eficiente el presupuesto empleado, también la relevancia de analizar previamente los contenidos a desarrollar, la competencia y principalmente el segmento al cual estará dirigido.

\section{REFERENCIAS BIBLIOGRÁFICAS}

De Benito, I. (2016). FACEBOOK Ads Instant Publicidad rentable en Facebook en 7 días. Plaforma: Iván De Benito

Del Santo, O. y Álvarez, D. (2012). Marketing de atracción 2.0 "como conseguir tus objetivos online con el mínimo presupuesto". Recuperado de https://www.juancmejia.com/wpcontent/uploads/2012/06/Marketing-de-Atraccion-20.pdf

Fundación Integra de Murcia. (2014). Tu plan de marketing en redes sociales: implantación y estrategias. Recuperado de https://www.cecarm.com/tu-plan-de-marketing-en-redessociales-implantacion-y-estrategias.pdf-6121?fbclid=IwAR1UCsHvSXz0f4dkvb9zy4mRvR7rR-iBrpUpT6CbYmIPi3-D-8NsT43a0U.

Gálvez, I. (2016). Facebook para empresas y emprendedores. México: IC Editorial.

Gamboa, R. (2017). APRENDE Facebook ads DESDE CERO. Plataforma: Roberto Gamboa.

Leal, A. y Quero, M. (2011). Manual de marketing y comunicación cultural. España: Proyecto Atalaya.

MUL-CANUL L. DEL C., PERALTA-MAZARIEGO E. R. GARCÍA-RODRÍGUEZ J. F. 
DESARROLLO DE UN PLAN DE SOCIAL MEDIA PARA POSICIONAR UNA EMPRESA EN CANCÚN, QUINTANA ROO.

Lecinski, J. (2011). ZMOT Ganando el momento cero de la verdad. Recuperado de https://www.juancmejia.com/wp-content/uploads/2012/06/Momento-Cero-de-la-VerdadZMOT-Google-Espa\%C3\%B1ol.pdf

Marquina, J. (2013). Plan social media y community manager. España: UOC.

Merodio, J. (2010). Marketing en redes sociales. Mensajes de empresa para gente selectiva. Recuperado de https://www.juancmejia.com/wp-content/uploads/2012/06/Marketing-enRedes-Sociales-Mensajes-de-empresa-para-gente-selectiva.pdf

Molina, G. (2013). Marketing en Facebook: Guía paso a paso para pymes. Recuperado de https://socialwithit.com/wp-content/uploads/2013/03/Social-With-It-Ebookcomo_convertirte_en_todo_un_especialista_en_marketing_en_facebook16.03.2013.pdf?fbclid=IwAR1Xkt9Gf8UAC6x5KYlg1Sol2DPRi1ksBf6gxrtCeyY_c08jL1r 7dyOlgWg

Moreno, R. (2017). Aprende Facebook ads paso a paso. Plataforma: Rosa Moreno

Peñarroya, M. (2014). Las oportunidades de las redes sociales para las pymes. Oikonomics: revista de economía, empresa y sociedad, (2), 20-24. Recuperado de http://oikonomics.uoc.edu/divulgacio/oikonomics/_recursos/documents/02/Oikonomics_ MPenarroya_es.pdf

Pérez, M. (2012). Redes sociales, mecanismos generadores de reputación organizacional para las PyMEs. Universidad \& Empresa, 14(22), 131-149. Recuperado de https://revistas.urosario.edu.co/index.php/empresa/article/view/2004/2050

Real, I., Leyva, A. y Heredia, J. (2014). Uso e impacto de las redes sociales en las estrategias de marketing de las PyME's. Revista de Investigación Académica Sin Frontera: División de Ciencias Económicas y Sociales, 7(19). Recuperado de http://revistainvestigacionacademicasinfrontera.com/inicio/wpcontent/uploads/2014/02/ Uso-e-impacto.pdf

Sierra. G. (2016). Facebook para negocios. México: Gerardo Sierra.

Smith, M. (2017). Marketing en Facebook: una guía completa para crear autoridad, generar compromiso y hacer dinero a través de Facebook. Recuperado de https://books.google.com.mx/books/about/Marketing_En_Redes_Sociales.html?id=mG v6uAEACAAJ\&redir_esc=y 
DESARROLLO DE UN PLAN DE SOCIAL MEDIA PARA POSICIONAR UNA EMPRESA EN CANCÚN, QUINTANA ROO.

Urueña, A., Ferrari, A., Blanco, A. y Valdecasa, E. (2011). Las redes sociales en internet. Recuperado de https://www.ontsi.red.es/ontsi/sites/ontsi/files/redes_socialesdocumento_0.pdf

Valle, A. (2015). Estrategias para el uso de un CRM. España: IT Campus Academy.

Wilcock, M. (2013). Marketing de contenidos. Recuperado de https://www.divisadero.es/wpcontent/uploads/publicaciones/Marketing-de-Contenidos2_para_web.pdf

\section{PÁGINAS WEB}

Comer en Cancún. (6 de junio de 2019a). Página de inicio [Publicación en Fanpage Facebook]. Recuperado de: https://www.facebook.com/ComerEnCancun/

Comer en Cancún. (6 de junio de 2019b). Estadísticas (Fanpage Facebook]. Recuperado de: https://www.facebook.com/ComerEnCancun/

Comer en Cancún. (6 de junio de 2019c). Página de inicio [publicación en Fanpage Facebook]. Recuperado de: https://www.facebook.com/ComerEnCancunOficial/

Comer en Cancún. (6 de junio de 2019d). Publicaciones [Fanpage Facebook]. Recuperado de: https://www.facebook.com/ComerEnCancun/ 